\title{
An Interactive Mobile Learning System for Generic Wireless Networks
}

\author{
S.W. Cheung, Jade Wong and W.T. Ko \\ Department of Electrical \& Electronic Engineering \\ The University of Hong Kong
}

\begin{abstract}
This paper describes how a simple interactive mobile learning system, originally developed for the $2 G$ system, evolved to a system for the 3G (TD-SCDMA) system, then to a system for the WiFi access system and finally become a generic Interactive Wireless Mobile Learning System which can be used in any wireless networks. The system designs in different stages of evolution are presented. To promote mobile learning and celebrate the Beijing 2008 Olympics, the Wireless Mobile Learning System was modified to become a tool used in an Inter-School Mobile Quiz Contest with participation of 17 secondary and primary schools and the results were very successful and encouraging.
\end{abstract}

Index Terms-E-learning technology, E-learning System, E-learning game, Interactive system, mobile system, wireless system, wireless applications

\section{INTRODUCTION}

Currently, the penetration of mobile subscribers in Hong Kong is more than $150 \%$ which means there are more mobile subscribers than the whole population. Mobile phone is becoming a necessity for many people who carry their mobile phones most of the time and spend a lot of time on talking to someone. Thus, few years ago, Cheung [1] had conceptualized the idea to design an interactive mobile learning tool so that mobile subscribers could use their mobile phones very conveniently to improve their learning in various subject areas such as Mathematics, Chinese or English, etc. anywhere and at anytime. As a result, a project to implement a simple wireless interactive game for use in mobile phones was proposed and then successfully developed by an undergraduate supervised by Cheung [1]. Due to the small screen sizes of mobile devices, difficulty in inputting texts and the limited speeds of the $2 \mathrm{G}$ systems, it was decided that the form of learning would be through questions of multiple choices and in all text-base. The game was later on called Wisdom in [1]. The technology used was simple and just a webbased system.

In 2007, the Hong Kong Wireless Technology Industry Association (HKWTIA) issued a Request for Proposals (RFP) on Mobile Services for "the Hong Kong Cyberport Time Division-Synchronous Code Division Multiple access (TD-SCDMA) Service Development Center". The RFP required that the proposed TD-SCDMA applications should be innovative and relating to the Beijing 2008 Olympic
Games. In respond to this RFP and based on the core idea of the Wisdom game, a project proposal entitled "TD-SCDMA Mobile Quiz Platform for the Beijing 2008 Olympic Games" was submitted and became one of the six successful awards [2]. TD-SCDMA is an international $3 \mathrm{G}$ standard developed by China and makes use of many advanced techniques such as uplink synchronization, smart antennas, joint detection, Baton handover, etc. to achieve high speed transmissions [3]. With the use of Time-Division Duplexing (TDD) to allow the uplink and downlink transmissions to time share the same carrier with allocation on a demand basis, TD-SCDMA is more suitable and efficient for internet access. Thus questions embedded with video clips could be used. With the experience gained from the Wisdoms game, Jade and her team were able to successfully complete the project in 3 months. The project was called the "3G Mobile Quiz Challenger" that could support a potentially large number of online players, depending on the capacity of the underlying network and its actual network traffic, to answer media-enriched, including video- or image-embedded, questions [4]. The 3G Mobile Quiz Challenger had been tested by the Datang Mobile on their TD-SCDMA network in China, and was demonstrated in the Olympic Experience Center in Beijing and the HKWDC in the Cyberport in Hong Kong. The project was also selected by the Hong Kong Trade Development Council as one of the exhibits in the Hong Kong pavilion in the PT/EXPO COMM CHINA 2007, the largest telecom exhibition in China. In the same exhibition, it was also selected by Datang Telecom as a $3 \mathrm{G}$ application showcase at the Datang Telecom pavilion.

Although the TD-SCDMA network is a high speed network, same as other $3 \mathrm{G}$ networks, the capacity is still limited. When questions embedded with pictures or video clips are sent over air to a large number of interactive players at the same time, congestion is unavoidable. Moreover, when using the $3 \mathrm{G}$ networks for data transmission, the charge is based on the volume of packets sent and received, thus it is costly for players to receive a huge number of data packets. One solution to the problem is to use a common broadcast channels, instead of dedicated channels, of the network for sending questions to players. However, this implies reallocation of the common broadcast channels and needs the agreement with the mobile network operators, 
putting a big constraint on the use of the $3 \mathrm{G}$ Mobile Quiz Challenger.

Many universities, including the University of Hong Kong, over the world are providing free $\mathrm{WiFi}$ access to all students using laptops, pocket PCs or WiFi-enabled mobile phones inside the campus, so we have used the same idea to design and develop an "Interactive Wireless Mobile Learning System" to facilitate interactive classroom quiz using mobile devices. With this system, students can access the system using mobile phones via the cellular mobile networks or using other WiFi-enabled devices via any WiFi access points in campus. A client interface program has been written in $\mathrm{J} 2 \mathrm{ME}$ for use in the wireless devices in the system. With this program installed on their WiFi-enabled devices such as PSPs, PDAs, WiFi-enabled mobile phones and laptops, students can simultaneously participate in an interactive on-line quiz during a lecture in a class room, or anytime and anywhere. Teachers can collect the student's answers immediately after each quiz question or test, obtain the distribution of the answers, and decide whether detailed explanations or additional lectures are necessary. Different flexible features have also been implemented. For example, the orders of quiz questions delivered to individual students could be in a random manner, preventing students from copying the answers from their neighbors.

The Beijing 2008 Olympics was a big event for China and Hong Kong which was a co-host city where the Equestrian Events were held. To promote mobile learning and to celebrate the Beijing 2008 Olympics, we organized an Inter-school Mobile Quiz Contest for the primary and secondary school students [5]. Based on Cheung's original concept, Jade was able to modify the Interactive Wireless Mobile Learning System to a tool for use in the contest within a very short period of time. There were a total of 17 schools, about 200 students and teachers, from all over Hong Kong, participating in the contest. In the contest, there were three question sessions, each having a specific theme, i.e., the Olympic Games, the Equestrian and The University of Hong Kong. The outcome was very successful.

It was argued that the computer was a prefect tool for learning [6]. However, as digital technologies have advanced so much and rapidly, we need to develop new learning environments that use the technologies to support learning. This paper presents one possible way.

The rest of this paper is organized as follows. The system descriptions and system architectures of the Wisdom game, the 3G Mobile Quiz Challenger and Wireless Mobile Learning System are described in sections 2, 3 and 4, respectively. The Inter-school Mobile Quiz Contest is described in section 5. Section 6 is Conclusions.

\section{WISDOM - A WIRELESS INTERACTIVE QUIZ GAME}

\subsection{System description}

Wisdom is a wireless and interactive game which could accommodate a large number of interactive players. To play the game, players were required to preregister with the game and had their mobile devices such as mobile phones or PDAs, etc. loaded with a client interface program written in J2ME. The players used their mobile devices to login the game via a $2 \mathrm{G} / 2.5 \mathrm{G} / 3 \mathrm{G}$ system in order to enter and then played the game. A number of quiz questions in the form of multiple choices in different subjects such as Mathematics, English, etc., were prepared for the game. In playing the game, all players received a set of about 30 questions in text one after the other, each having 4 possible answers as multiple choices. Players selected the correct answers from these choices within a limited period of time, say, 30 secs. If they selected the correct answer, they stayed on; otherwise they lost and were gone. The one who correctly and continuously answered the most quizzes was the winner. Since players could access the game via the internet anywhere and at anytime, it was possible that they could be competing against tens or hundreds of players simultaneously. This made the game much more interesting and exciting than the traditional mobile quiz games.

To make the game even more interesting, before answering each question, each player was given 3 options to choose: Option 1) "Skipping", i.e. if the player was not sure about all the answers, he/she could select this option to skip the question; Option 2) "50:50", i.e. if the player was not sure about the some of answers given, by selecting this option, two incorrect answers $(50 \%)$ out of the 4 given were removed so the player had a higher chance to answer the quiz correctly; and Option 3) "Prolonging the Time Limit" which doubled the time limit to $60 \mathrm{sec}$. so that the player could have more time to answer the current question. All players would only have a limited number, say 5 times out of 30 questions, of having this option. At the end of each question, the correct answer was displayed on their mobile devices, thus they could learn from this.

\subsection{System architecture}

The wisdom system consisted of the following components:

a) a game web server;

b) a number of mobile phones installed with a J2ME client interface program; and

c) an administration console;

The system architecture of wisdom is shown Fig. 1. Players use their mobile handsets installed with a client interface program to access the game server via a $2 \mathrm{G} / 2.5 \mathrm{G} / 3 \mathrm{G}$ mobile network. The flow of the game is 
shown in Fig. 2. At the beginning of the game, the server waits until all clients have logged and then prompts all the clients with a question in the form of multiple choices. When the answers from all players are received, the server checks the returned answers against the correct ones stored in the database and sends the correct answer to the clients. The players with wrong answers are stopped from playing the game further. When there is only one left in the game, it is the winner.

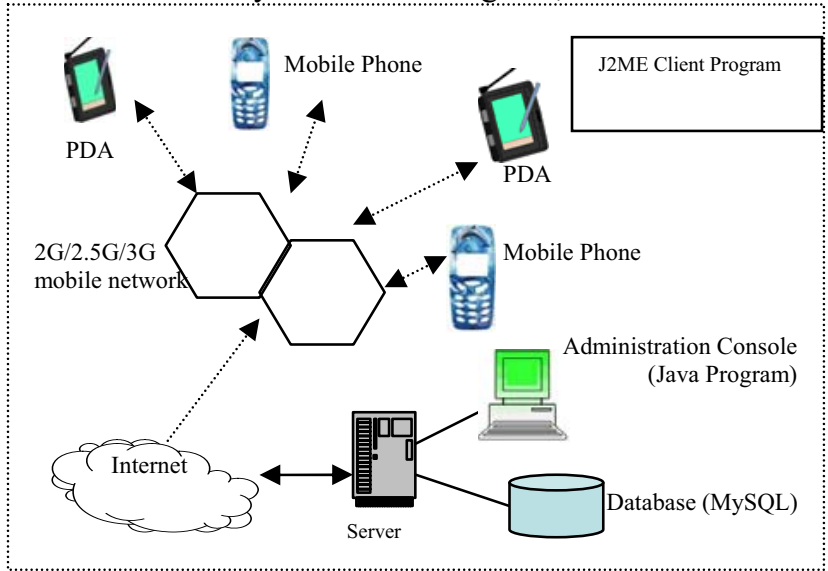

Figure 1 System architecture of Wisdom

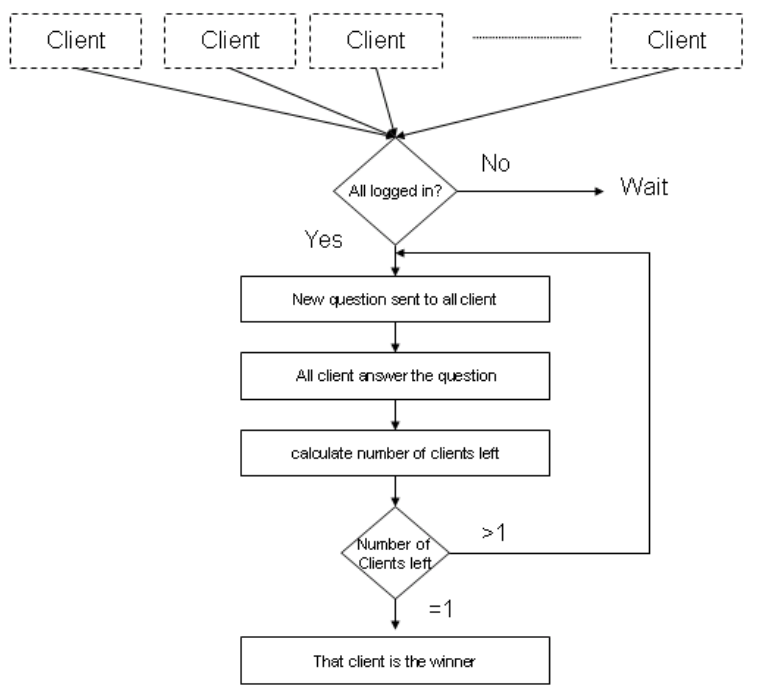

Figure 2 Game flow of Wisdom

\section{THE 3G Mobile Quiz Challenger}

\subsection{System description}

Same as the Wisdom game, players of the game were required to register with the system before they could download and execute an interface program written in J2ME. Players first selected a desire language (English or Chinese) and then input the Login user IDs and passwords. We had prepared more than 20 Olympic Game events in the system and players could join any one of them to play.

The game was designed to be played during the breaks of the selected Olympic Game event such as the
Aquatics - Diving. It was expected that there could be hundreds or even thousands of players in each session. When the game session started, players would all be prompted with the same quizzes one-by-one simultaneously in the form of multiple choices. Some quizzes were purely texts, but some were with pictures or video clips for the main reason that the TD-SCDMA system could support high speed data transmissions. All quizzes were relating to the Olympic Games or Chinese history which were part of the objective of the project. To play the game, players simply answered each quiz by selecting one of the choices displayed on their mobile phones. Before answering the question, each player was given 3 options: Option 1) "Skipping", Option 2) "50:50" and Option 3) "Prolonging the Time Limit". The meanings of these options were exactly the same as those used in the Wisdom game. Each player could use this option three times within a game session. Each player was given 5 times to use this option. If the players finished using these 5 times, they had to play the game all by themselves. The players selecting the correct answer stayed in the game and they were prompted with another quiz. However, the players selecting the wrong answers were kicked out from playing the game. They became observers. The correct answer for each question displayed on the mobile phones at the end of each question. The one who stayed last in the game was the winner. If there was more than one player who was able to select all the correct answers, then there would be more than one winner. A congratulation message, description of the award and also the award instructions was sent to the winners in the form of SMS/ MMS via the SMS Gateway and displayed on their mobile phones. All the top 10 winners in each session were awarded with souvenirs. The awards were sent as e-coupons displayed in the mobile phones for the winners to redeem the souvenirs from the Olympic souvenir shops.

The names of the winners in each game session were also be published on a Mobile Quiz Game Portal so that individual player could check this using their web browsers on their mobile phones or from any nearby PC terminals.

\subsection{System architecture}

The 3G Mobile Quiz Challenger was modified from the Wisdom game and consisted of the following components:

a) the Mobile Quiz Game Portal (customized for the Beijing 2008 Olympic Games during the pilot phase);

b) TD-SCDMA based Mobile Quiz Game Platform Server;

c) Administration Console Portal (a web portal);

d) SMS Gateway.

The system architecture of the 3G Mobile Quiz Challenger is shown in Fig. 3. There was a T1 lease line connecting the server to the test TD-SCDMA network in 
Beijing dedicated for the project. All players of the game again were pre-registered and have their TDSCDMA mobile phones loaded with a client interface program written in J2ME. (At that time, there were only very few TD-SCDMA mobile phones available for use and we were given two of them for use in the project.) The players logged on the Quiz Game Platform Server via the TD-SCDMA network through the client interface of the mobile phones. The quiz questions, answers, and players' information were all stored in the database files managed through the MySQL server. Since the clients' returned answers had relatively smallsized data packets, the game server could accommodate hundreds or thousands of pre-registered users' requests in an efficient manner without suffering from the performance bottleneck.

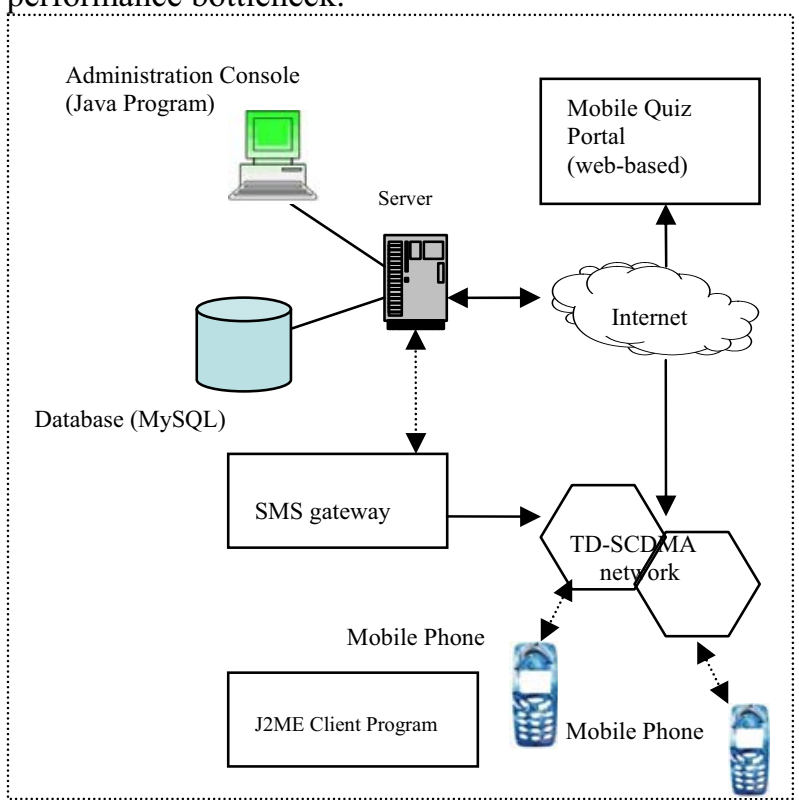

Figure 3 System architecture of 3G Mobile Quiz Challenger

\section{INTERACTIVE WIRELESS MOBILE LEARNING SYSTEM}

\subsection{System description}

The Interactive Wireless Mobile Learning System was modified from the $3 \mathrm{G}$ Mobile Quiz Challenger and designed to be used in classes to facilitate interactive classroom learning. Students logged on the system using their mobile devices such as $2.5 \mathrm{G}$ and $3 \mathrm{G}$ mobile phones via the mobile networks, or WiFi-devices such as WiFi-enabled mobile phones, laptops, PSP or PDAs etc. via any access points in the campus. To use this online system, students were required to pre-register in the particular courses in which their teachers wished them to participate. They were given the usernames and passwords to log on the system.

The teachers prepared the quiz questions in the form of multiple choices beforehand and stored them with answers in the database server. An easy interface program had been developed for this purpose. To monitor the progress of the students during the lecture, the teacher, if wished, could use the system to send each of the prepared questions one-by-one to the students' wireless devices and asked the students to answer. Having received all the answers back from all students (which was checked by the system), the teacher used the system to display the distribution of the answers for the question and hence decided whether more detailed explanations or additional lectures were required for a particular topic of the course.

Different features had also been implemented. For example, the teacher could prepare a whole set of questions and used it as a formal test. In that case, a time limit was set to allow the students to complete the test. The order of the questions sent to the individual students could be in a random manner, so that students could not copy the answers from their neighbors. At the end of the test, the teacher could obtain the score of each student immediately.

\subsection{System architecture}

The system architecture of the Interactive Wireless Mobile Learning System is very similar to that of the $3 \mathrm{G}$ Mobile Quiz Challenger system. The main difference is the SMS gateway which is no longer needed in the Interactive Wireless Mobile Learning System.

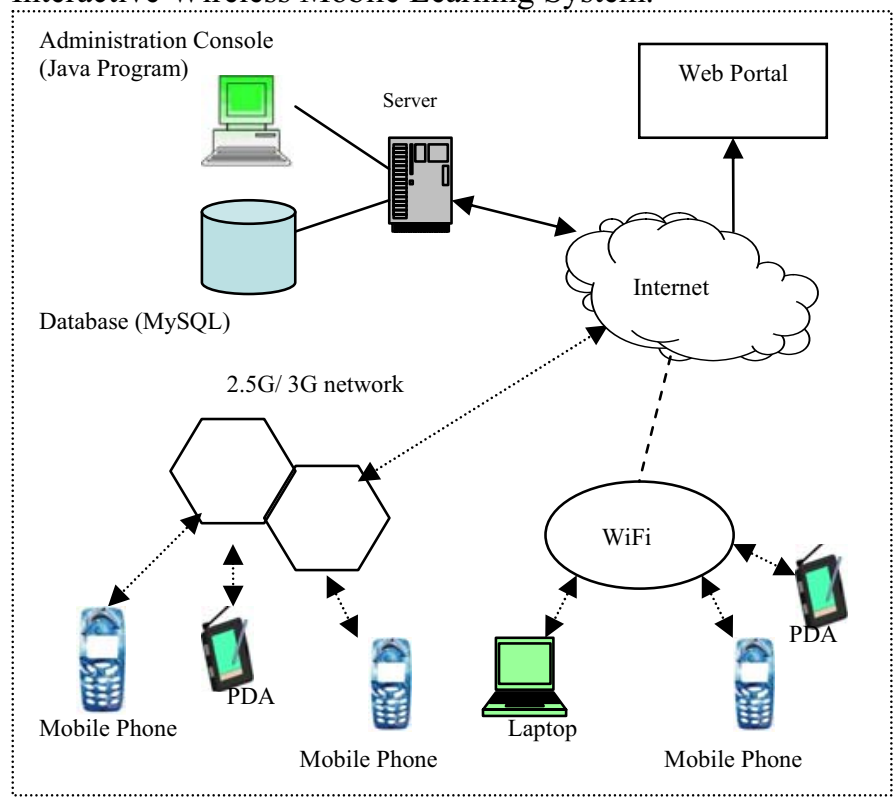

Figure 4 System architecture of Interactive Wireless Mobile Learning system

Figure 4 shows the system architecture which consists of the following components:

a) A Mobile learning platform server.

b) Administration Console Portal (a web portal).

c) A number of WiFi Access points.

d) A display program to display the percentage of correct answer for each questions and marks scored by each student. 
Students used their mobile devices installed with an interface program, their usernames and their passwords to access the system server via any WiFi access point in the campus or theoretically any mobile networks (which should be seldom used due to the high cost). For security reasons, the MAC addresses of the WiFienabled devices were also needed to register in order to gain access to the $\mathrm{WiFi}$ access points in the campus.

\section{INTER-SCHOOL MOBILE QUIZ CONTEST SYSTEM}

\subsection{System description}

The idea for the Inter-school Mobile Quiz Contest was to promote mobile learning and celebrate the Beijing 2008 Olympics. We modified the Interactive Wireless Mobile Learning System to a tool to be used in this contest. There were a total about 200 students and teachers from 17 secondary and primary schools participating in the contest which was held in a hall of about 250 seats. The contest was divided into three quiz question sessions, with each having a specific theme. They were about knowledge on the Olympic Games, the Equestrian and The University of Hong Kong. Each school nominated 4-6 students as the contestants to participant in each question session. These students were pre-registered with the system. In each session, there were about 25-30 quiz questions in the form of multiple choices and students were give 5-10 mins to answer all of them. The quiz questions could be purely texts, with or without pictures. The Students were given the same type of WiFi-enabled device, a Sony PSP, to access the system via three different access points to answer the quiz questions. The contestant who could answer most questions was the Champion of the session. During the quiz session, the scores for each of the contestants were displayed on a big screen in real time so that their supporters could see. This made the contest a very exciting event.

\subsection{System architecture}

The system architecture of the Inter-school Mobile Quiz Contest System is shown in Fig. 5 which is very similar to but simpler than that of the Interactive Wireless Mobile Learning system. It consists of a server and a client program for accessing the server. Students used the PSPs with the client program installed, their usernames and passwords to access the system via the WiFi access point in the quiz sessions.

All the answers, quiz questions and users' information were stored in relational database file formats managed through the MySQL server. The server kept track of the returned answers from each contestant, checked them against the actual answers in the data base, computed the score and displayed them on the screen in real time. At the end of each quiz question session, we could display the distribution and the performance of each contestant.

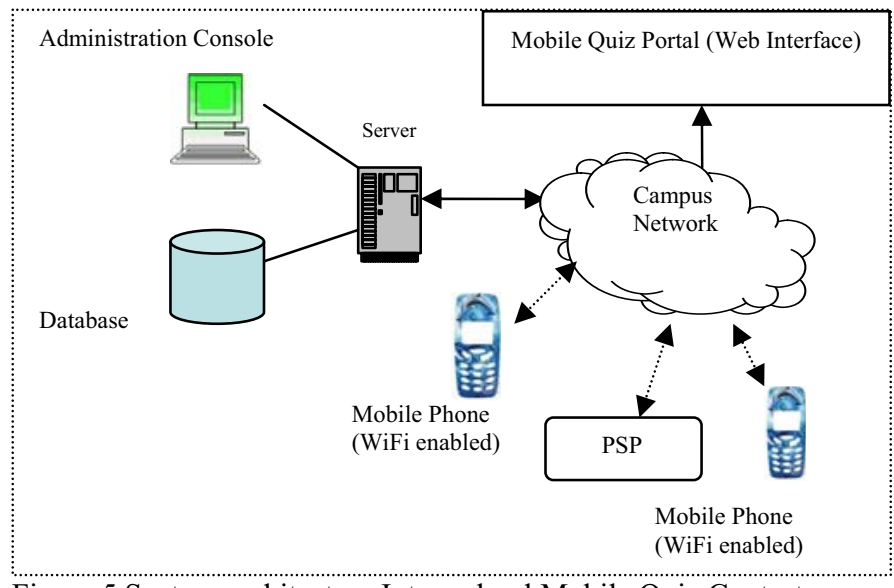

Figure 5 System architecture Inter-school Mobile Quiz Contest System

At the end of the contest, very good comments were received from teachers and students. Students found it very interesting and teachers found it very useful on monitoring the learning progress of students.

\section{CONCLUSIONS}

In this paper, we have presented the system description and system architecture of a simple interactive mobile learning system, Wisdoms, original developed for the $2 \mathrm{G}$ system. The system evolved to the 3G Mobile Quiz Challenger system used in a $3 \mathrm{G}$ system (TD-SCDMA) and then to an Interactive Wireless Mobile Learning System to be used in any wireless networks. The system description and system architecture in each stage of evolution have been described. To promote mobile leaning and celebrate the 2008 Beijing Olympics, the system was modified to a tool used in an Inter-school Mobile Quiz Contest. Very good comments were given by the students and teachers.

\section{REFERENCES}

[1] W.T. Ko, "Education Software", Final-Year Project Report. 2007, The University of Hong Kong

[2] Proposal: "TD-SCDMA Mobile Quiz Platform for the Beijing 2008 Olympic Games" for the Mobile Services of the "Hong Kong Cyberport TD-SCDMA Service Development Center" 2007

[3] Freescale Semicondutor, Application Notes, Introduction to TD-SCDMA on the MRC6011 RFF Device, Rev. 1, $11 / 2004$

[4] About the 3G Mobile Quiz Challenger DOCI= http://olympic.eee.hku.hk

[5] About the Mobile Quiz Contest. DOCI= http://www.eee.hku.hk/achievements/achievements/mlea rning_8July.htm

[6] R.B. Kozma, The implications of cognitive psychology for computer-based learning tools, Educational Technology, 27(11), 20-25, 1987. 